\title{
Are Runners More Prone to Become Infected with COVID-19? An Approach from the Raindrop Collisional Model
}

\author{
Francisco J. Arias ${ }^{1}$ \\ Received: 1 May 2020 / Accepted: 9 July 2020 / Published online: 5 August 2020 \\ (c) Beijing Sport University 2020
}

\begin{abstract}
It is known that COVID-19 spread mainly from person-to-person through respiratory droplets produced when an infected person coughs or sneezes, and as a result certain ideas about contagious of COVID-19 have been spread. One of them is the widespread belief that close runners, owing to the stronger exhalation, can be more prone to be infected with COVID-19 because the collision with the suspended respiratory droplets should the runner in front be infected. However, because of the low Stokes number this idea cannot be generalized without carefully thought and in fact can be put into question. Utilizing the raindrop collisional model and with the help of computational fluid dynamics (CFD), it is shown that the probability of collision with respiratory droplets is not always increasing with the approaching velocity of the runner but rather there is a maximum velocity threshold at which the efficiency of collision drops.
\end{abstract}

Keywords COVID-19 contagious; airbone $\cdot$ Spread diseases

\section{Introduction}

It is known that COVID-19 spread mainly from person-toperson through respiratory droplets with diameters around $\approx 5 \mu \mathrm{m}$ or thereabouts, which are produced when an infected person coughs or sneezes [4].To date, vaccine is not available, and as a result exceptional protection measures are being taken in the affected countries such as maintaining at least 1-2 $\mathrm{m}$ distance between persons and/or wearing masks in places prone to concentration of people. By keeping a safe distance between persons, it is pretended that tiny droplets has enough time to fall to the ground under gravity and then with a limited distance for transmission [2-8]; nevertheless, more recent studies seem to indicate that even $2 \mathrm{~m}$ of inter-personal distance could not be enough [5]. On the other hand, there is the widespread belief that close runners, because the stronger exhalation, can be more prone to

Electronic supplementary material The online version of this article (https://doi.org/10.1007/s42978-020-00071-4) contains supplementary material, which is available to authorized users.

Francisco J. Arias

francisco.javier.arias@upc.edu

1 Department of Fluid Mechanics, Polytechnic University of Catalonia, ESEIAAT C/Colom 11, 08222 Barcelona, Spain be infected with COVID-19 because the collision with the suspended respiratory droplets should the runner in front be infected.

\section{Statement of the Model}

Let us consider Fig. 1 in which a runner infected with COVID-19 carries the virus and leaving behind a trail of contaminated cloud with suspended respiratory droplets containing the virus. At the same moment a second healthy, non-infected runner is approaching the contaminated cloud with a certain velocity. Computational models have been recently used considering a similar problem and considering saliva droplets with average diameters around of $80 \mu \mathrm{m}$, [2]. However, no mechanistic models based on collisions probability have not yet reported.

\section{Raindrop Collisional Model}

The mechanistic model for raindrops falling from the sky and growing during their path by a collisional process with other tiny drops encountered during the travel is a wellgrounded theory in cloud physics and can be found in fundamental books on the topic, see for example [6] and [3]. The raindrop collisional model is based on the calculation 


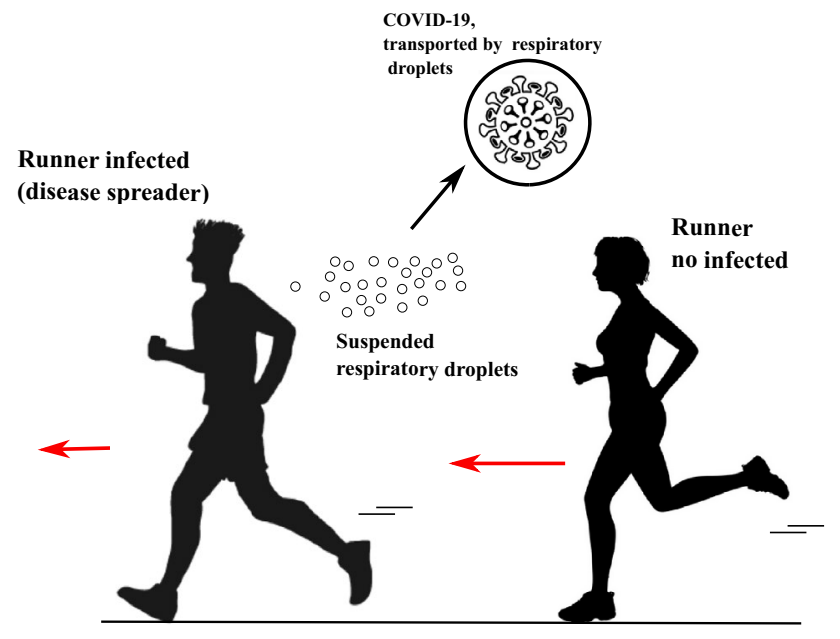

Fig. 1 A healthy non-infected runner approaching a suspended contaminated COVID-19 cloud left by the runner in front

of an effective collisional cross-section as depicted in Fig. 2, which is summarized as follows. In order for a falling drop (the collector drop) to collide with a second stationary drop (the collected drop), it must be inside of a certain area which is less than the geometric area because the air streamlines bowing out around the collector drop carry the smaller drops with them around the drop, and the effective cross-section becomes less than the actual cross-section. As drops get bigger, they have too much inertia to follow the air streamlines, thus making the collision more likely. This fact is typified by the Stokes number Stk, which is a dimensionless number characterizing the behavior of particles suspended in a fluid flow. For the purpose of the present study, it is enough to know that when the Stokes number is much smaller than unity Stk $\ll 1$, a body suspended in air will follow the air streamlines closely (perfect advection) [7], i.e., the path followed by the body is the same as the air streamlines. For the application of our case of study dealing with respiratory droplets with diameters $\approx 5 \mu \mathrm{m}$ and then with a very small Stokes number, the assumption of perfect advection is reasonably justified.

The most important parameter within the raindrop collisional theory is the collision efficiency, $E$ which is defined as [6]

$E=\frac{r^{2}}{\left(R+r_{d}\right)^{2}}$

where $R$ and $r_{d}$ are the radius of the collector and collected drop, respectively (see Fig. 2). When $R \gg r_{d}$ as is our case of study, Eq. (1) simplifies as
$E \approx \frac{r^{2}}{R^{2}}$

\section{Discussion}

It is interesting to apply the raindrop collisional model for our case of interest. To do this, some idealizations and simplifying assumptions are required.

Firstly, for preliminary assessment, we model the runner as a cylinder with a radius $R$ of infinite length and then neglecting disturbances of the flow due to end effects as depicted in Fig. 3.

Secondly, because of the very low Stokes number, we assume perfect advection and then the tiny respiratory droplets follow streamlines. Thus, the collision of droplets with the runner is reduced to the calculation of air streamlines surrounding the cylinder and with a collision efficiency $E$ calculated from Eq. (2) as is depicted in Fig. 4.

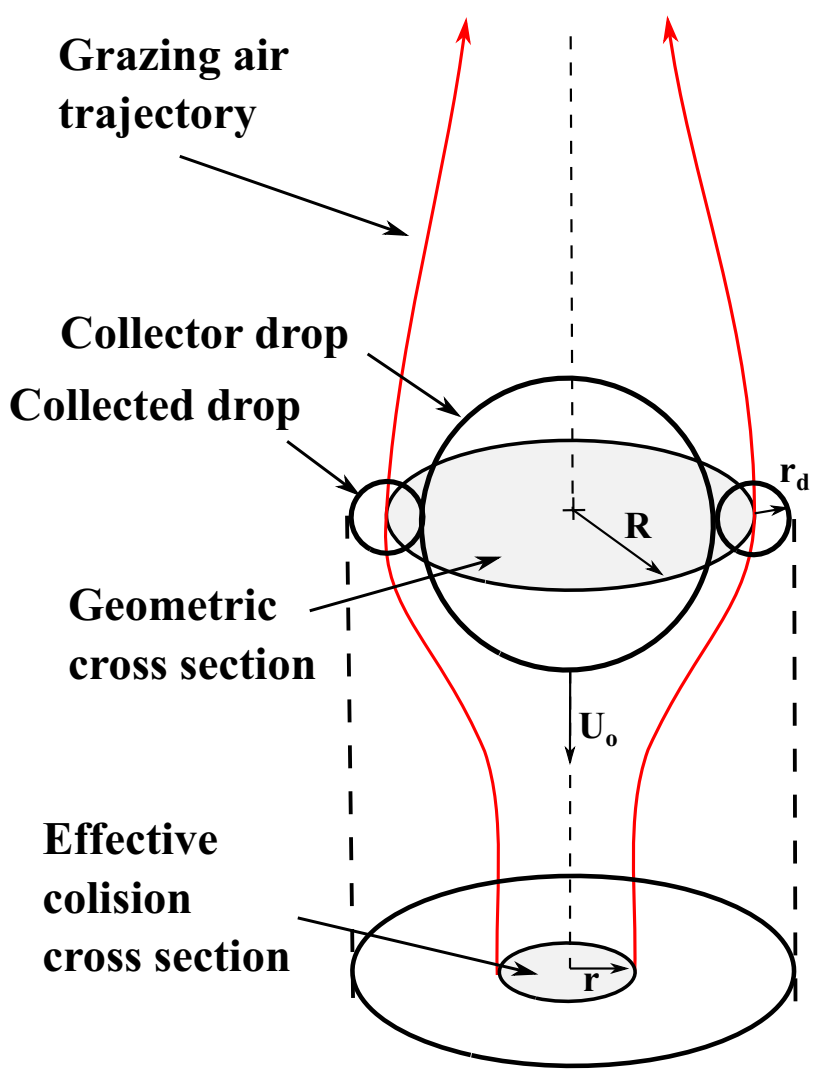

Fig. 2 Air flow around a falling particle. Only the air in innermost streamline collides with the particle, the rest goes around it. Credit. Lamb and Verlind [3] 

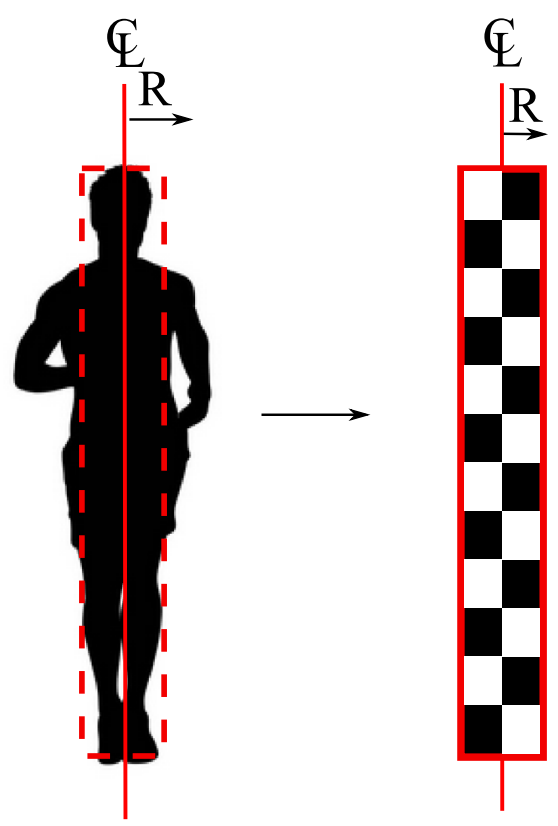

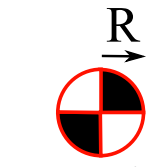

Top view
Fig. 3 Actual shape of the physical model

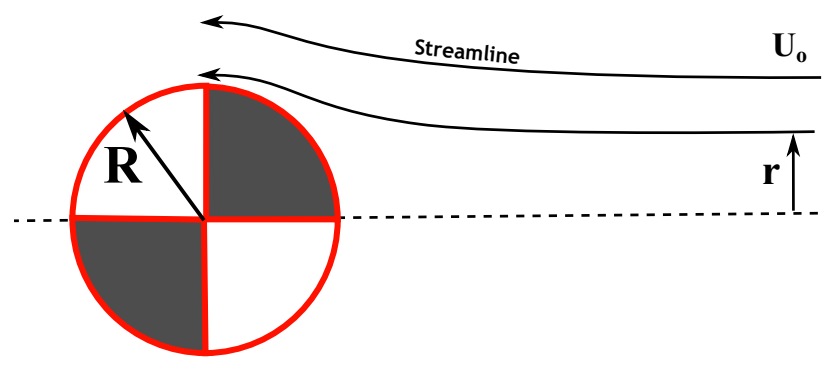

Fig. 4 Aerodynamic trajectories of the droplets. Because the very small sizes of the droplets, complete advection can be assumed and then drops travel following streamlines

\section{Results}

To obtain the estimation of the collision efficiency as a function of the approaching velocity, computational fluid dynamics (CFD) simulations were performed using the ANSYS-CFD code FLUENT. The fluid simulated was air and the properties were taken as constant an at room temperature $T=293 \mathrm{~K}$ and taking the parameters for simulation by default including the K-epsilon (k-e) turbulence model as the most common model used. For the simulations, a cylinder with an equivalent radius $R=5.5 \mathrm{~cm}$ was assumed. The resulting curve is shown in Fig. 5. It is interesting to see that the collection efficiency has a peak of efficiency which actually is very small with a peak around a 3\% and with approaching velocities around $0.6 \mathrm{~m} / \mathrm{s}$ or thereabouts which is justified by the advection of the tiny respiratory micro-particles with the stream lines. This velocity is much more close to average walkers rather than runners where as seen in the figure the collision efficiency drops.

\section{Conclusions}

The probability of collision between a runner and micrometric respiratory droplets suspended in the air and at rest (from environment) was discussed within the framework of a raindrop collisional model. It was shown that, as expected from this theory, the probability of collision is not increasing indefinitely with the approaching velocity of the runner but rather there is a maximum peak or threshold velocity after which the efficiency of collision drops.

It must be stressed that the present work must be taken with caution. Substantial uncertainties were present at every step of the analysis. The probability of collision reported result from unavoidable idealizations which are inherent in any theoretical model and in special in the raindrop collisional model and therefore the results are not intended to typify quantities. This should not be misconstrued as an attempt to produce a definitive mechanistic analysis. Nonetheless, the raindrop collisional model provides an interesting alternative approach which has been applied successfully in cloud physics for the growth of raindrops in meteorological situations which by far are much more complex and difficult to accurately predict in comparison with our case of study. All in all, the present work will provide important guidance in future efforts to analyze the problem and considering all the situations.

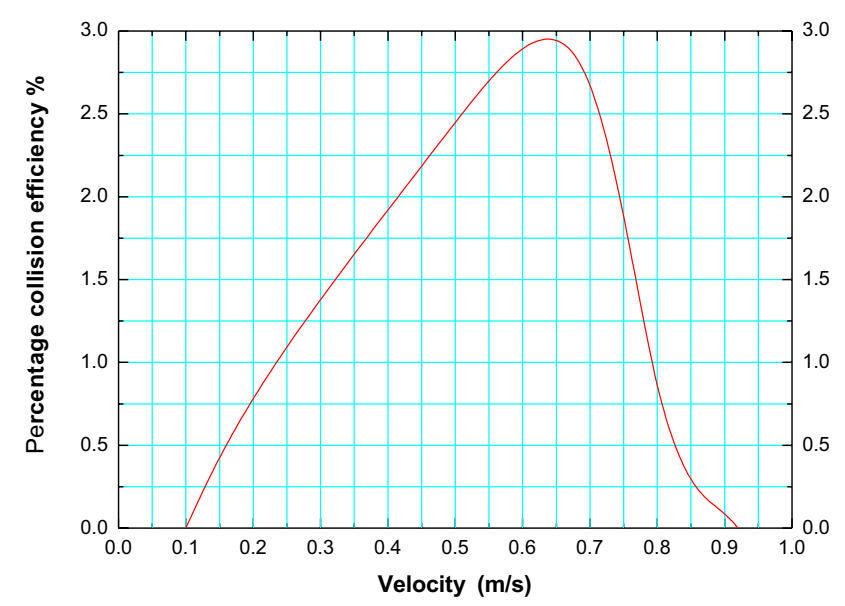

Fig. 5 Collision efficiencies for COVID-19 virus considering a 5- $\mu \mathrm{m}$ respiratory droplet as a function of the approaching velocity 
Acknowledgements This research was supported by the Spanish Ministry of Economy and Competitiveness under fellowship grant Ramon y Cajal: RYC-2013-13459.

\section{References}

1. Atkinson J, Chartier Y, Pessoa-Silva CL, Jensen P, Li Y, Seto WH. Natural ventilation for infection control in health-care settings. Geneva: World Health Organization; 2009.

2. Blocken B, Malizia F, van Druenen T; Marchal T. Towards aerodynamically equivalent COVID19 $1.5 \mathrm{~m}$ social distancing for walking and running. 2020. Pre-print Available online: http:// www.urbanphysics.net/Social\%20Distancing\%20v20_White _Paper.pdf. Accessed 14 April 2020.

3. Lamb D, Verlinde J. Physics and chemistry of clouds. Cambridge: Cambridge University Press; 2011.
4. Li H, Liu SM, Yu XH, Tanga SL, Tang CK. Coronavirus disease 2019 (COVID-19): current status and future perspective. Int J Antimicrob Agents. 2020 (in press).

5. Setti L, Passarini F, Gennaro G, Barbieri P, Perrone MG, Borelli M, Palmisani J, Gilio AD, Piscitelli P, Miani A. Airborne transmission route of COVID-19: why 2 meters/ 6 feet of inter-personal distance could not be enough. Int J Environ Res Public Health. 2020;2020(17):2932.

6. Rogers RR. A short course in cloud physics. Oxford: Permagon; 1976.

7. Tropea C, Yarin A, Foss J. Springer handbook of experimental fluid mechanics. Berlin: Springer; 2007.

8. Xie X, Li Y, Sun H, Liu L. Exhaled droplets due to talking and coughing. J R Soc Interface. 2009;6:703-14.

9. Zhu SW, Kato S, Yang JH. Investigation of SARS infection via droplets of coughed saliva. Built Environment and Public Health, Proceedings. 2nd International Conference on Built Environment and Public Health. 2004. pp. 341-354. 